\title{
Insole optical fiber Bragg grating sensors network for dynamic vertical force monitoring
}

Maria Fátima Domingues

Cátia Tavares

Cátia Leitão

Anselmo Frizera-Neto

Nélia Alberto

Carlos Marques

Ayman Radwan

Jonathan Rodriguez

Octavian Postolache

Eduardo Rocon

Paulo André

Paulo Antunes 


\title{
Insole optical fiber Bragg grating sensors network for dynamic vertical force monitoring
}

\author{
Maria Fátima Domingues, ${ }^{\mathrm{a}, \mathrm{b}, \mathrm{c}, \star \star}$ Cátia Tavares, ${ }^{\mathrm{a}}$ Cátia Leitão, ${ }^{\mathrm{a}, \mathrm{c}}$ Anselmo Frizera-Neto, ${ }^{\mathrm{d}}$ Nélia Alberto, ${ }^{\mathrm{a}, \mathrm{e}}$ \\ Carlos Marques, ${ }^{\mathrm{a}, \mathrm{c}}$ Ayman Radwan, ${ }^{\mathrm{a}}$ Jonathan Rodriguez, ${ }^{\text {, }}$ Octavian Postolache, ${ }^{\mathrm{f}}$ Eduardo Rocon, ${ }^{\mathrm{b}}$ \\ Paulo André, ${ }^{g}$ and Paulo Antunes ${ }^{a, c}$ \\ anstituto de Telecomunicações, Campus Universitário de Santiago, Aveiro, Portugal \\ ${ }^{b}$ Centro de Automática y Robótica, CSIC-UPM, Arganda del Rey, Madrid, Spain \\ 'University of Aveiro, Department of Physics and I3N, Campus Universitário de Santiago, Aveiro, Portugal \\ ${ }^{\mathrm{d}}$ Federal University of Espírito Santo, Department of Electrical Engineering, Goiabeiras, Vitória, Brazil \\ eUniversity of Aveiro, Centre for Mechanical Technology and Automation, Department of Mechanical Engineering, Aveiro, Portugal \\ 'Lisbon University Institute, ISCTE-IUL, Instituto de Telecomunicações, Lisbon, Portugal \\ gUniversity of Lisbon, Instituto Superior Técnico, Instituto de Telecomunicações and Department of Electrical and Computer Engineering, Lisbon, \\ Portugal
}

\begin{abstract}
In an era of unprecedented progress in technology and increase in population age, continuous and close monitoring of elder citizens and patients is becoming more of a necessity than a luxury. Contributing toward this field and enhancing the life quality of elder citizens and patients with disabilities, this work presents the design and implementation of a noninvasive platform and insole fiber Bragg grating sensors network to monitor the vertical ground reaction forces distribution induced in the foot plantar surface during gait and body center of mass displacements. The acquired measurements are a reliable indication of the accuracy and consistency of the proposed solution in monitoring and mapping the vertical forces active on the foot plantar sole, with a sensitivity up to $11.06 \mathrm{pm} / \mathrm{N}$. The acquired measurements can be used to infer the foot structure and health condition, in addition to anomalies related to spine function and other pathologies (e.g., related to diabetes); also its application in rehabilitation robotics field can dramatically reduce the computational burden of exoskeletons' control strategy. The proposed technology has the advantages of optical fiber sensing (robustness, noninvasiveness, accuracy, and electromagnetic insensitivity) to surpass all drawbacks verified in traditionally used sensing systems (fragility, instability, and inconsistent feedback). () 2017 Society of Photo-Optical Instrumentation Engineers (SPIE) [DOI: 10.1117/1.JBO.22.9.091507]
\end{abstract}

Keywords: gait vertical ground reaction force; gait plantar pressure; physical rehabilitation; optical fiber sensors; insole fiber Bragg gratings network.

Paper 160820SSPR received Nov. 30, 2016; accepted for publication Feb. 8, 2017; published online Feb. 28, 2017.

\section{Introduction}

The continuous aging of the population and lifetime expectation increase entail health risks that require a close monitoring of elder citizens and patients. With the witnessed demographic shift in the world's population allied to a growing sedentary lifestyle, the demand for remote, continuous, and dynamic healthcare monitoring systems to enhance the quality of life is increasing. ${ }^{1}$ Such solutions should enable debilitated people to sustain a fulfilling lifestyle by monitoring and controlling their physical incapacities. ${ }^{2}$

Aiming to contribute toward enhancing the life quality of physically debilitated and elder citizens, this work proposes an optical fiber sensors' network solution adaptable into an insole for vertical ground reaction forces (VGRF) and plantar pressure monitoring. The assessment of these parameters is of great importance for the gait analysis health evaluation. Additionally, by mapping the ground reaction forces during gait, it is possible to understand the effect induced in the body. ${ }^{3-5}$

Gait represents the movement of the body center of mass (BCM) through a horizontal trajectory and it implies, in a

*Address all correspondence to: Maria Fátima Domingues, E-mail: fatima. domingues@ua.pt normal gait cycle, that the feet alternate in their ability to sustain the body posture, by placing one foot forward followed by the next. ${ }^{6}$ Therefore, gait is a cycle activity that can be divided into two main phases: stance, when the foot is in contact with the ground and swing, when such contact ceases. The stance phase starts when the heel strikes (HS) the ground, lasting until the moment the toe becomes the last contact point [toe off (TO)]. The swing phase starts when the toe ceases its contact with the ground and lasts till the instant the HS the floor again, the point at which a new gait cycle starts. For normal individuals, walking at a normal pace, the stance phase corresponds to about $\sim 62 \%$ of the entire gait cycle. ${ }^{4}$

Gait analysis can be referred to as the systematic examination of the locomotion, and it can be used in the evaluation of pretreatment, for surgical decisions and postoperative assessment of patients. ${ }^{4}$ The distribution of the VGRF and plantar pressure on the surface of the foot during its contact with the ground provides valuable information regarding abnormal posture shifts, the spinal cord well-being, as well as the foot structure and condition. ${ }^{7}$ Also, there are pathologies that can be related to an abnormal VGRF and plantar pressure, such as the high risk for ulceration in diabetic patients with high forefoot plantar pressure

1083-3668/2017/\$25.00 @ 2017 SPIE 
during gait. $^{8}$ Therefore, the accurate monitoring of the VGRF and plantar pressure distribution is vital to avoid and reduce the risk of such pathologies.

Additionally, for the rehabilitation robotics field, the development of a flexible insole pressure sensor, to measure and analyze force distribution on a patient's foot, would represent a real breakthrough. The possibility of having this information directly from a sensor could dramatically reduce the computational burden of exoskeletons' control strategy because currently it is estimated based on very complex biomechanical models. ${ }^{9}$

The dynamic and continuous monitor of gait parameters requires that the sensing mechanisms implemented are mobile, with limited or nonexistent wiring, preferably adaptable to a shoe, low cost, and with low power consumption. ${ }^{10}$ Although a considerable number of solutions for plantar pressure have been already reported, they are mainly based on electronic or imaging devices, presenting some drawbacks such as fragility, instability, and inconsistent feedback. ${ }^{10,11}$

As an alternative to these electronic devices, optical-fiberbased sensors offer a smaller (diameter of several hundred micrometers), robust, minimally invasive, biocompatible, highly accurate, electromagnetic insensitive, electrical isolation (no electrical power at the measuring point), and a multiplexable solution, allowing several sensors into the same fiber cable. ${ }^{12,13}$ The optical fiber technology is widely spread and used to monitor parameters, such as pressure, temperature, humidity, strain, refractive index, angular displacements, and acceleration, among others. ${ }^{12-19}$ Also, optical fiber sensing technology has already been used to monitor static VGRF and plantar pressure values. ${ }^{20-22}$ Nevertheless, to date, no reports on dynamic continuous measurements during gait have been presented. ${ }^{21,22} \mathrm{On}$ that note, the achievement of our work regarding the dynamic and continuous optical data acquisition during gait is of great significance for the field.

The proposed optical system architecture to monitor VGRF consists of a network of fiber Bragg grating (FBG) sensors incorporated into an insole, matching the sensing requirements mentioned before (mobile, with limited wiring, adaptable to a shoe, low cost, and low power consumption), allied to the high accuracy and all the advantages of optical fiber sensing.

The FBGs can be described as periodic perturbations of the refractive index of the fiber core along the longitudinal axis of the optical fiber. The grating structure can be induced by an ultraviolet (UV) laser, which is used to create a selective wavelength reflective structure that follows the Bragg condition. ${ }^{14,15}$ The sensing principle relies on the monitoring of the wavelength reflected by the inscribed grating ( $\lambda_{B}$-Bragg wavelength) and its variation as a function of the parameter under study (e.g., strain and/or temperature). The Bragg wavelength shift, $\Delta \lambda_{B}$, can be related to strain $(\Delta \varepsilon)$ and/or temperature $(\Delta T)$ variations by the following:

$\Delta \lambda_{B}=S_{\varepsilon} \times \Delta \varepsilon+S_{T} \times \Delta T$,

where $S_{\varepsilon}$ and $S_{T}$ are the sensor sensitivities to strain and temperature variations, respectively. The first term is associated to the strain influence on $\lambda_{B}$, whereas the second term represents the temperature effect in the same parameter. ${ }^{16}$

After this initial introduction, in the following section (Sec. 2), the description of the insole design, production, assembling, and calibration are presented. Section 3 deals with the results and analysis of the data acquired during the testing of the developed solutions for VGRF monitoring. Finally, the main conclusions retrieved from this work are pointed out in the Sec. 4.

\section{Insole Development and Production}

In this section, the development and calibration of a new platform based on the optical fiber sensing technology to monitor the VGRF and plantar pressure during gait are presented. This solution can be used as a fixed platform or as an insole adaptable to a shoe.

\subsection{Insole Incorporation of the Fiber Bragg Grating Network}

The optical platform is composed of a cork sole, with $1.0-\mathrm{cm}$ thickness, in which FBG sensors were incorporated in critical points of analysis [heel, midfoot, metatarsal, and toe areas, Fig. 1(a)] to monitor the VGRF, ${ }^{10,23}$ as shown in Figs. 1(b) and $1(\mathrm{c})$.

The cork was the material chosen to embed the sensors due to its excellent properties for this application, namely thermal isolation, malleability, and a near-zero Poisson ratio. ${ }^{24}$ The first is extremely relevant, once the body temperature may influence the FBGs sensing feedback. By using a thermal isolation material, no compensation is needed in the acquired Bragg

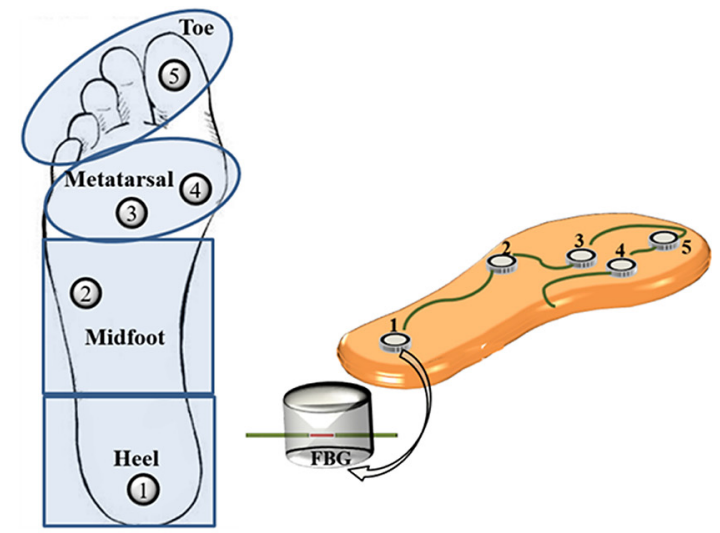

(a) (b)

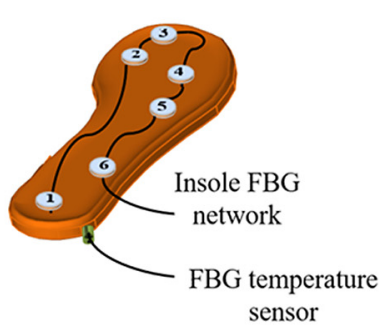

(c)

Fig. 1 Schematic representation of (a) the foot plantar main areas, (b) the sensors network implemented for a fixed platform, and (c) the sensors network implemented for the insole adapted in a shoe. 


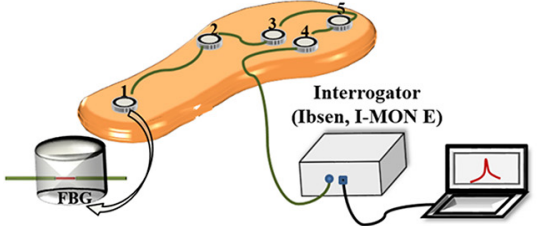

(a)

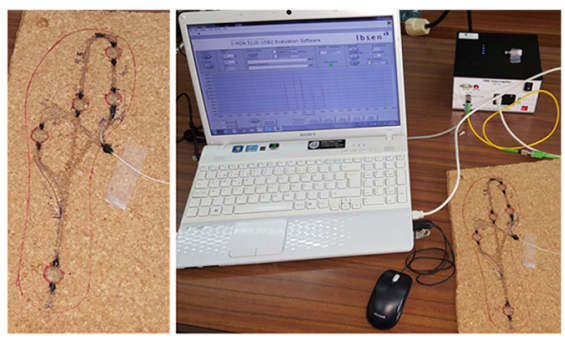

(b)

Fig. 2 Fixed platform monitoring system: (a) schematic representation and (b) photograph.

wavelength. Also, the malleable properties of the cork make it easy to incorporate the sensing elements in the required points of analysis, which additionally facilitates the sole implementation in any type of shoe configuration. Moreover, and although malleable to handle, it also provides the necessary resistance to prevent the optical fiber network to be damaged or broken during the gait movement. Finally, its near-zero Poisson ratio is an advantage to its use, once the pressure applied in one area of the plantar surface will not influence the other areas, allowing in that way to isolate the pressure points, preventing sensor's cross talk. $^{24}$

The FBG sensing elements were inscribed and multiplexed into a GF1-photosensitive optical fiber (Thorlabs ${ }^{\circledR}$ ) cable, using an UV KrF Bragg Star ${ }^{\mathrm{TM}}$ Industrial-LN pulsed $\mathrm{KrF}$ excimer laser operating at $248 \mathrm{~nm}$, applying pulses with energy of $5 \mathrm{~mJ}$ and repetition rate of $500 \mathrm{~Hz}$. Phase masks customized for 248-nm UV laser were chosen for the 1550-nm spectral region grating inscription (Ibsen Photonics). In order to protect and provide extra resistance to the optical fiber sensing elements, the FBGs were encapsulated in epoxy resin (Liquid Lens ${ }^{\mathrm{TM}}$ ) cylinder structures $(1.0-\mathrm{cm}$ diameter and $0.5-\mathrm{cm}$ height). Each sensing element consists of such cylindrical epoxy structure with the FBG at the middle position. The entire FBG sensing network (epoxy structure included) was incorporated within the cork structures, in order to obtain a compact (no air gap within it) insole.

Two solutions were developed, one to be used as a fixed platform [Fig. 1(b)] containing five FBG-based sensors and another to act as an instrumented insole to be adapted into a shoe [Fig. 1(c)] with six FBG sensors. The second solution was intended to be able to monitor all the critical points ${ }^{10,23}$ for VGRF analysis, but due to equipment restrictions, only six points were considered.

For the insole, an FBG temperature sensor was also inserted into the cork near the first sensing point. This procedure was implemented to guarantee that the sensing points are not influenced by any temperature change, and if they are, it can be properly compensated.

\subsection{Monitoring System Assemble and Calibration}

For the monitoring and calibration of both systems, the FBG sensing network was connected to a portable interrogation system constituted of a battery, a miniaturized broadband optical ASE module (B\&A Technology Co., As4500), an optical circulator (Thorlabs, 6015-3), and an optical spectrometer (Ibsen, I-MON 512E-USB). The latter operates at a maximum rate of $960 \mathrm{~Hz}$, with a wavelength resolution of $5 \mathrm{pm}$, responsible for the acquisition of the Bragg wavelength shift, which is proportional to the vertical force applied on the sensing element points, as shown in Fig. 2.

Prior to its implementation for VGRF determination, the developed platform and insole were calibrated. This procedure was made with one load sequence on each isolated sensor. The contact area was of $1.0 \mathrm{~cm}$ (diameter of the loading prove used during the calibration process). For that purpose, different load values, ranging from 0 to $170 \mathrm{~N}$, in $10-\mathrm{N}$ steps increment, were induced in the FBG sensing elements, using a Shimadzu ${ }^{\circledR}$ AGS-5 kND mechanical test machine. The reflected Bragg wavelength shift of each FBG in the network was registered for each load value. The calibration values for the five sensing points in the fixed platform are presented in Fig. 3.

A linear dependence of the Bragg wavelength shift with the applied loads was obtained, with sensitivity coefficients ranging from $3.38 \mathrm{pm} / \mathrm{N}$ (FBG 4) to $11.06 \mathrm{pm} / \mathrm{N}$ (FBG 2). The differences in the strain sensitivities may be related to the methodology used to incorporate the FBGs in the insole. As the cork used is a porous material, different amounts of the epoxy resin may have infiltrated into its surroundings. Also, although major effort was made to keep all the FBGs at the same depth of the cylinder epoxy resin, some deviations might have occurred, due to the resin's initial liquid properties. Nonetheless, such facts do not influence the obtained final results, once all the sensing elements were independently calibrated. In future works, special attention will be given to this aspect.

The sensitivity coefficients, for each FBG sensor, in the platform and insole implementations, were later applied to retrieve

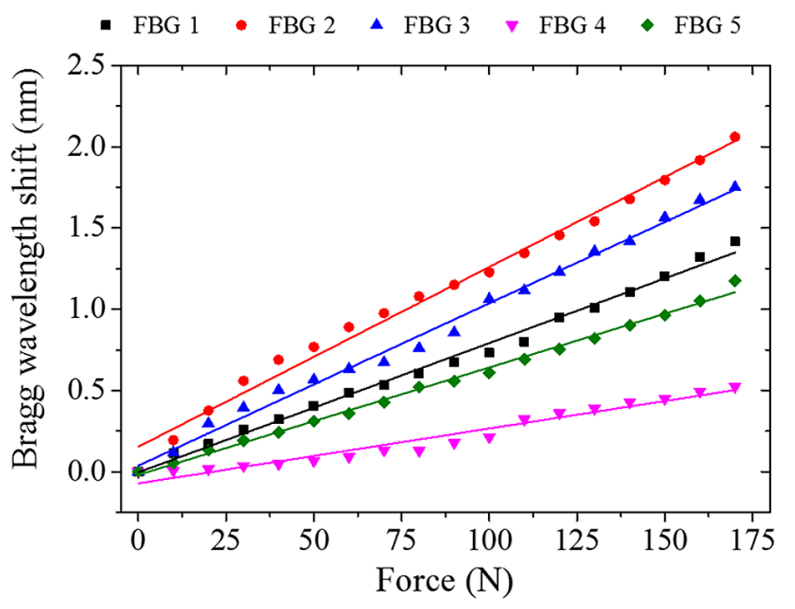

Fig. 3 Bragg wavelength shift as function of the applied vertical force. Points are correspondent to the experimental data and the lines to the linear fits $\left(0.953<R^{2}<0.995\right)$. 
the vertical force value from the acquired Bragg wavelength shift data.

\section{Vertical Ground Reaction Forces Platform Testing and Insole Integration}

After the calibration, three sets of studies were implemented in order to verify the reliability of both the fixed platform and the insole developed.

\subsection{Platform Testing}

The VGRF induced in the sensing elements during a normal gait movement was analyzed with the platform fixed at the ground, as shown in Fig. 4. The response of each sensing element to the VGRF during a gait cycle was repeated and acquired five times. The feedback of the platform to the displacement of the BCM was also evaluated.

In Fig. 5, the acquired data are presented, from which it is possible to verify that the sensing network response is similar for the five passages, confirming the repeatability of the sensor's response.

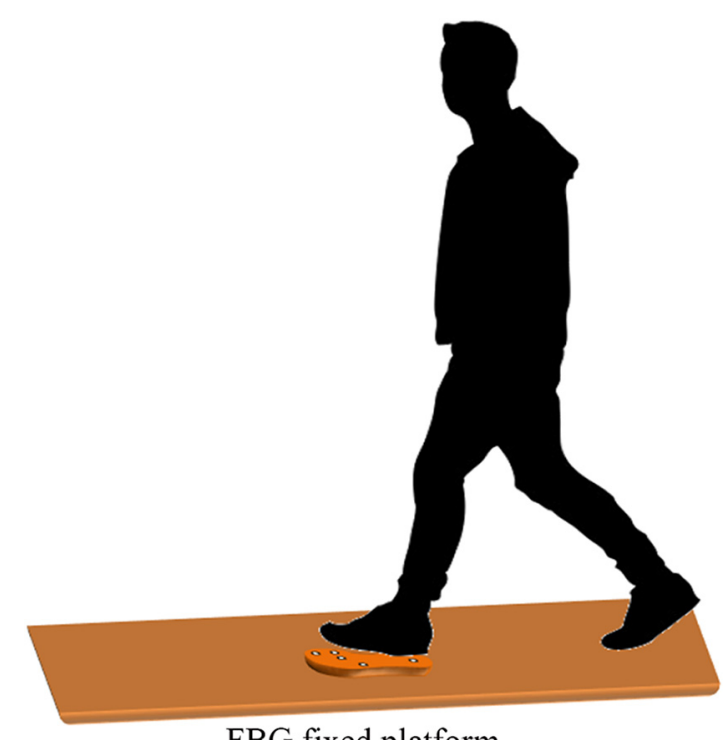

FBG fixed platform

Fig. 4 Schematic diagram of the protocol implemented for gait analysis using the fixed platform.

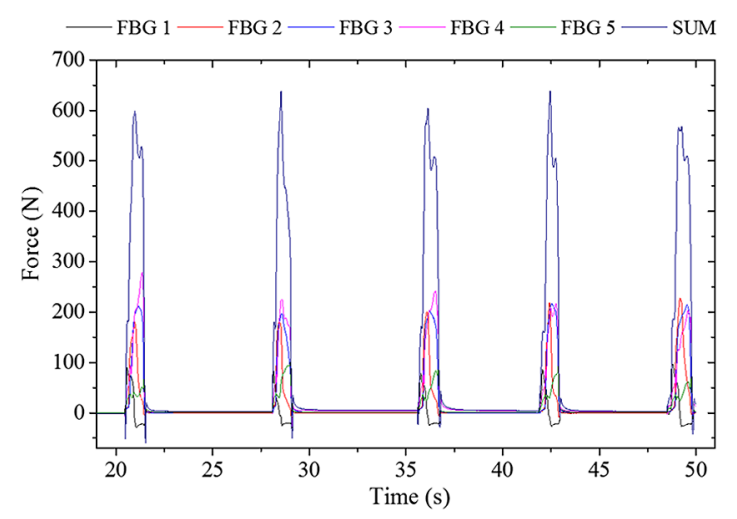

Fig. 5 VGRF obtained during the five steps and the resulting curve of all the sensors response sum for each step.
A detailed view of the stance period of the gait cycle is shown in Fig. 6. It is possible to retrieve the sequence in which the sensors are activated and the VGRF associated at each point under analysis. The observed maximum amplitude obtained for each sensor was temporally registered according to what is expected in a gait movement, ${ }^{4}$ verifying a typical VGRF curve during the stance period of the gait cycle. ${ }^{4}$

The stance period is initiated when the heel touches the ground, HS; after the contact of the heel with the ground, the foot is moved toward a stable support position for the body in which the hip joint becomes aligned with the ankle joint; this phase is called midstance phase (MS). The purpose of the following movements is to propel the BCM forward, which is initiated by the rise of the heel (HR) and its loss of contact with the ground. In this movement, the last point of contact of the foot with the ground should be the toe at the moment known as the TO, which also corresponds to the end of the stance phase in a gait cycle. ${ }^{4}$

According to the layout of the sensing network, it can be observed that the beginning of the HS moment is marked by the FBG1 with greater response, which is expected, as this sensor is placed at the heel. This response is maintained until the MS stage (in which all the sensors are activated) finishes. It can also be verified that at the HR's initial moment, the VGRF values registered for the sensor FBG 1 considerably drop, and the most accentuated forces are registered for the sensors placed in the metatarsals areas (FBG 2, 3, and 4). Also, at this moment, the VGRF registered at the toe location by the sensor FBG 5 becomes more accentuated and hits its maximum as the toe becomes the last point of support in the stance phase at the TO stage.

From the positive feedback of the fixed platform during the performed tests, it becomes evident that the method implemented is an adequate solution for VGRF monitoring during gait. Moreover, from the analysis of the forces registered during the stance phase, it is also possible to infer and monitor the plantar pressures of individuals. ${ }^{4,10,23}$

The BCM displacements, in the body sagittal and frontal planes of motion, were also analyzed using the same platform. For that purpose, an 85-kg male, was asked to place his foot on the sensing platform and to execute a series of BCM movements (with a 5-s duration each), starting by standing still with the $\mathrm{BCM}$ centered $(\mathrm{C})$, followed by an anterior $(\mathrm{A})$ position and

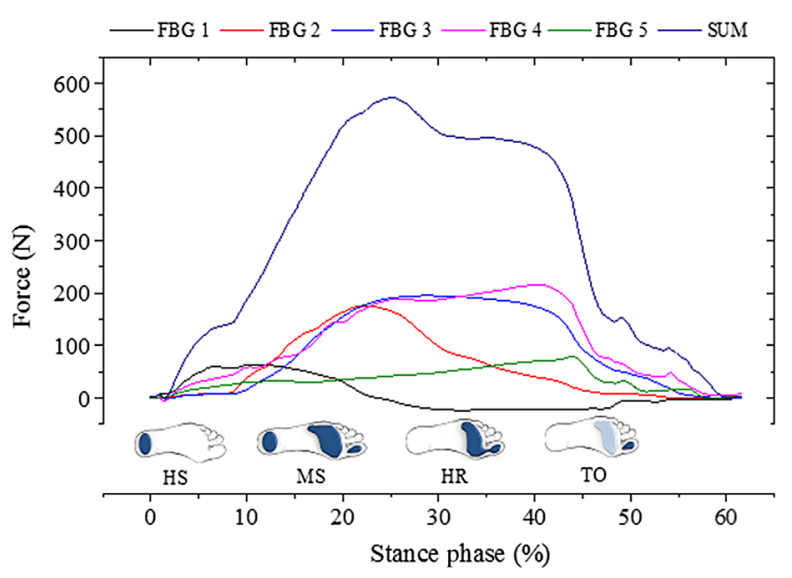

Fig. 6 Average VGRF obtained during the stance period (62\%), from the individual steps analyzed (the regions of the foot touching the platform in each gait step are in dark blue). 


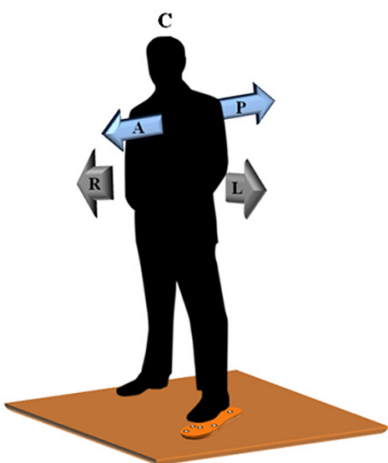

(a)
1. Standing still with the $\mathrm{BCM}$ centered, position $\mathrm{C}$

2. Sagittal displacement to the position $A$

3. Back in position $\mathrm{C}$

4. Sagittal displacement to the position $P$

5. Back in position $\mathrm{C}$

6. Frontal displacement to the position $L$

7. Back in position $\mathrm{C}$

8. Frontal displacement to the position $\mathbf{R}$

9. Back in position $\mathrm{C}$

Fig. 7 (a) Schematic diagram of the protocol implemented for the analysis of the BCM displacement (sagittal and frontal displacements in blue and gray, respectively) and (b) descriptive protocol (the subject remained in each position for $5 \mathrm{~s}$ ).

then back to the original position (C) from which goes to posterior $(\mathrm{P})$ position and then resting again at the center $(\mathrm{C})$. After the sagittal displacement, a frontal displacement was executed, in which the subject moved the BCM first to the left (L), back in the center $(\mathrm{C})$ and then to the right $(\mathrm{R})$, and finally back in the center (C). In Fig. 7, the implemented protocol is schematized.

During the protocol implementation, the Bragg wavelength shift induced in the sensing network was acquired and the corresponding VGRFs were retrieved. Figure 8 presents the response of each sensor, during the different moments of the tests performed.

In the anterior movement, an increase of the vertical forces registered by the sensors positioned in the metatarsal and toe areas is evident, while the sensor placed in the heel section indicates a decrease of the VGRF. On the other hand, during the posterior displacement of the BCM, the VGRF at the heel area is more accentuated, while the force values at the toe and metatarsal areas decrease.
Regarding the centered position of BCM (C), the areas that are mostly actuated in the platform are the metatarsal and midfoot areas, indicating that those zones are the more pressed ones in the ground in the sustainment of the subject's body weight, while standing.

The behavior monitored by the FBGs network is within the expected; hence, in the anterior movement of the BCM, the body weight will be mainly distributed by the metatarsal and toe areas, while in the posterior movement, it will be mostly supported by the heel area.

Regarding the frontal plane displacements, the sensors located at the extremities of the platform (FBG 2 and 4) should be the ones presenting a higher variation of the VGRF. In fact, it is noticeable that during such movements, the sensor placed in position 2 registers the increase of vertical force, when the BCM is displaced to the left, while the sensor placed in position 4 shows a similar behavior when the BCM is moved to the right. Regarding the sensors positioned at the central of the metatarsal

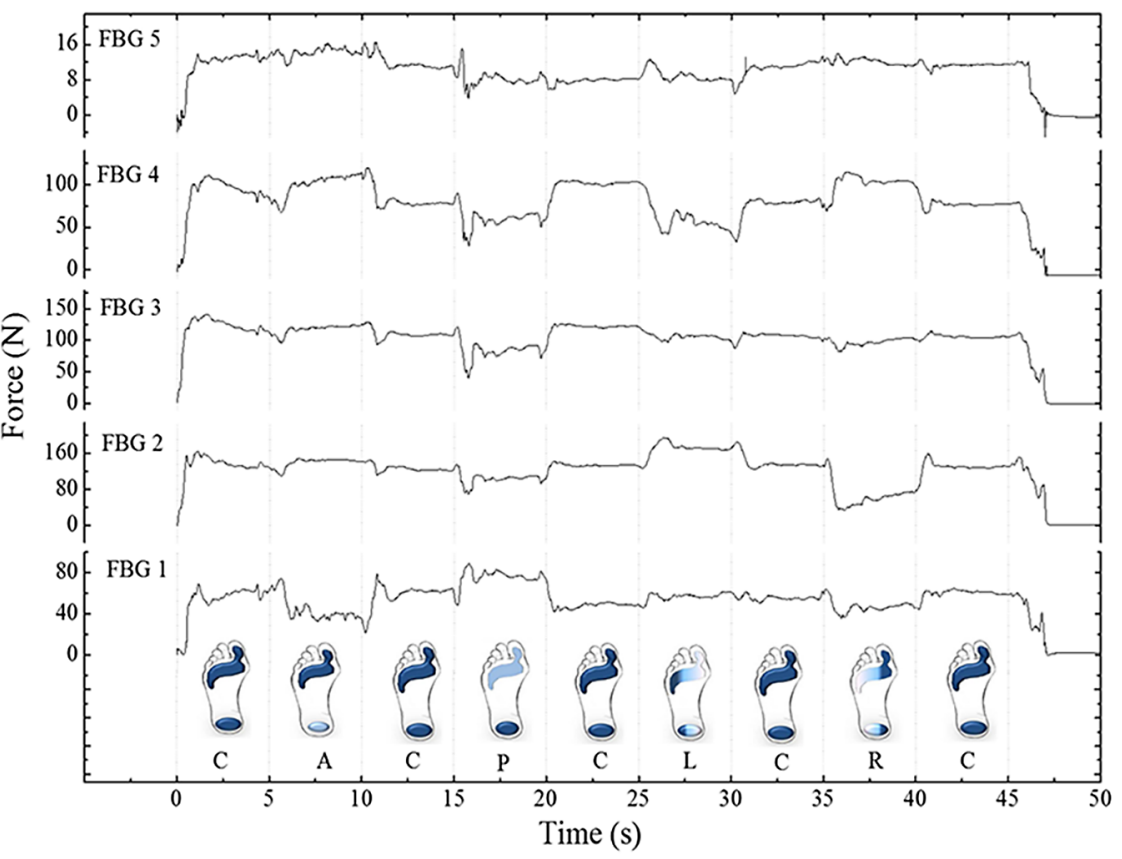

Fig. 8 Representation of the vertical forces detected during the BCM displacements [the VGRF intensity on each foot location is colored, in the scheme, from light (less intensity) to dark (more intense) blue]. 
area, the value registered through the frontal protocol implementation is mostly constant, which indicates that in such BCM displacement, the vertical force is mostly shifted between the left and the right metatarsal areas.

\subsection{Instrumented Shoe}

In order to perform a dynamic and autonomous VGRF monitoring during gait, an insole with six FBG sensors network was designed and adapted to a shoe, as shown in Fig. 9.

In addition to the sensing network for VGRF monitoring, an FBG temperature sensor ${ }^{16}$ was also adapted to the insole in order to monitor the temperature variations and guarantee that the Bragg wavelength shift registered during the gait cycles is only the result of the VGRF actuating on the sensing elements. In fact, due to the cork thermal isolation characteristics, ${ }^{24}$ it is expected that the body temperature will not influence the Bragg wavelength shift of the sensing network, once its sensing elements are placed within the cork sole and not in direct contact with the body. The wavelength shift values obtained for the temperature sensor confirms what was previously stated, as its value remained constant during the gait cycles experiments, as presented in Fig. 10.

Regarding the gait cycles analysis, the subject, in this case, a 45-kg female, was instructed to walk in a normal pace, during which the Bragg wavelength was acquired by the interrogation system. In Fig. 10, the vertical forces, registered in the insole sensing network for three gait cycles, are presented, in addition to the temperature recorded during the test.

Similar to the behavior registered in the fixed platform (Sec. 3.1), the elements initially actuated are the ones placed within the heel area (FBG 1 and 6), followed by the metatarsal area (FBG 5, 4, and 2) and ending with the element within the toe area (FBG 3) at the conclusion of the stance phase.

Also, from the gait cycles monitoring, the different phases in the gait cycle, namely the stance phase that characterizes $\sim 68 \%$ of the gait cycle for the subject tested, and the swing phase, during which the foot is not in contact with the ground, are clearly

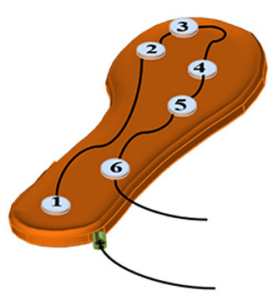

(a)

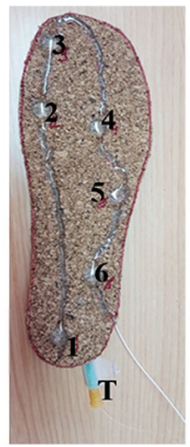

(b)

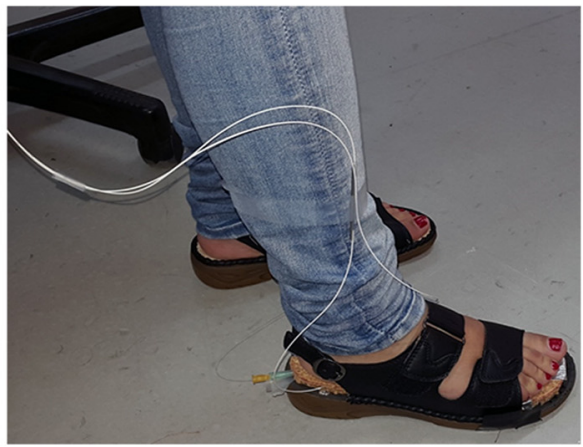

(c)

Fig. 9 (a) Schematic representation of the designed insole, (b) photograph of the insole with the sensing network and temperature sensor, and (c) photograph of the instrumented shoe.

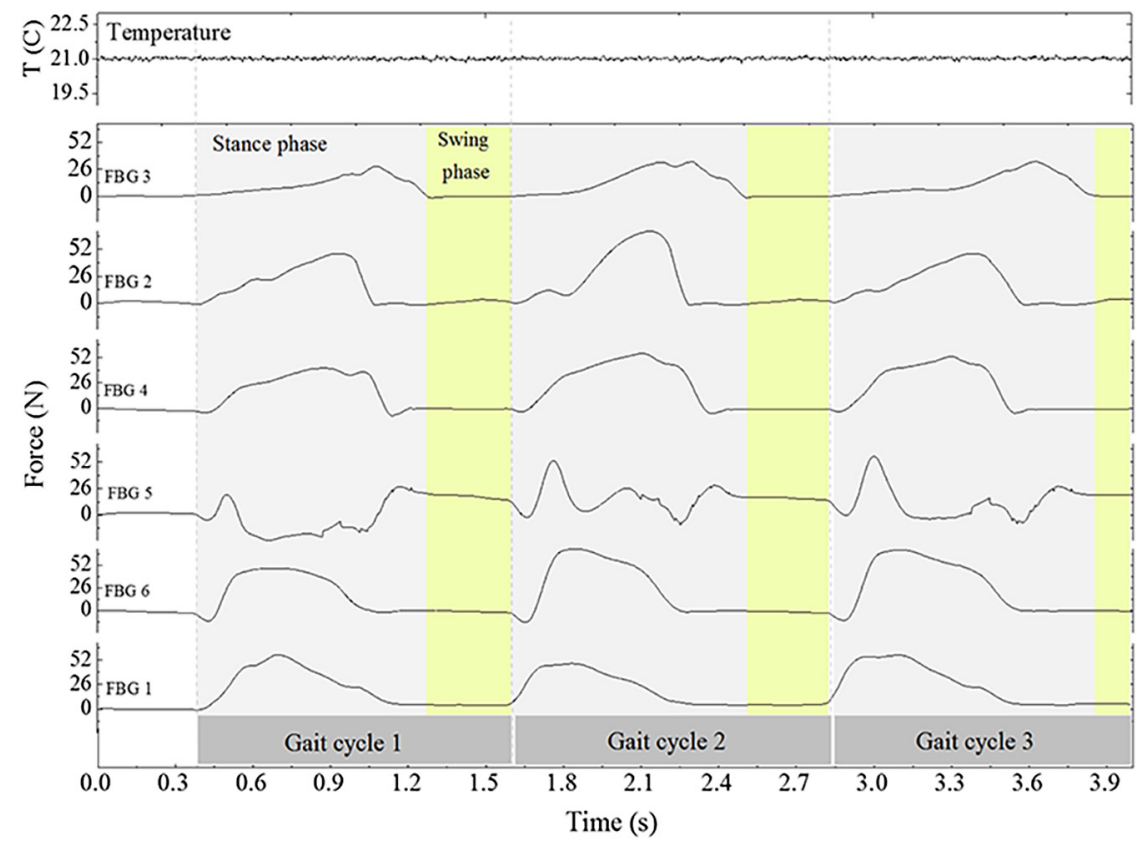

Fig. 10 Temperature and the vertical forces registered in the insole sensing network for three gait cycles. 
distinguished. During the latter phase, the VGRF is not present, and therefore, the Bragg wavelength shift of all the sensing elements is mostly constant during that phase.

\section{Conclusion}

In this work, an optical fiber sensing architecture to monitor the VGRF induced in the foot plantar surface during gait and BCM displacements is presented. The paper shows the design and implementation of a platform with a five FBGs network, placed in key points to monitor the VGRF on the foot surface during gait cycles and the BCM displacements. Also, an insole instrumented with a six FBGs network was designed and implemented within a shoe for dynamic monitoring of gait.

The calibration and laboratory measurements for both solutions are presented. The results obtained demonstrate the accuracy and reliability of the proposed systems to monitor the VGRF during the tests implemented. Moreover, due to the reduced size and resilience of the sensing network, the proposed solutions are a noninvasive tool to monitor the vertical forces induced on the foot plantar surface without impairing the mobility of the user.

The analysis of the vertical forces distribution can provide important data toward the foot plantar pressure mapping, which enables inferring vital information with regards to the foot structure and health condition, in addition to the detection of any anomaly related to the spinal function. Moreover, such plantar pressure mapping can be also applied in the detection of high-risk alerts related to pathologies, such as the foot ulceration in patients with diabetes. Additionally, the development of a flexible insole pressure sensor for the rehabilitation robotics field would represent a real advance in the monitor and analysis of a patient's foot force distribution allowing to considerably reduce the computational burden of exoskeletons' control strategy, currently based on very complex biomechanical models. The proposed noninvasive solution represents a step forward in the field of e-Health toward providing continuous and close monitoring of impaired patients and/or elder citizens, while maintaining their mobility and without compromising their life quality or their daily activities.

\section{Disclosures}

The authors have no relevant financial interests in the paper, and no other potential conflicts of interest to disclose.

\section{Acknowledgments}

This work was funded by FCT/MEC through national funds and when applicable cofunded by FEDER - PT2020 partnership agreement under the projects UID/EEA/50008/2013 and UID/ CTM/50025/2013. The work was also supported from funding by FCT PTDC/DTP-DES/6776/2014 and Instituto de Telecomunicações through the project WeHope. Maria Fátima Domingues, Nélia Alberto, and Carlos Marques acknowledge the financial support from FCT through the fellowships SFRH/ BPD/101372/2014, SFRH/BPD/78141/2011, and SFRH/BPD/ 109458/2015, respectively. Cátia Tavares acknowledges the financial support from WeHope project (ref. 818/2016). Ayman Radwan acknowledges the financial support from FCT through the researcher grant (ref. IF/01393/2015). Anselmo Frizera-Neto acknowledges CAPES (88887.095626/2015-01) and FAPES (67566480 and 72982608). Eduardo Rocon acknowledges CAPES (PVE no A126/2013). We also acknowledge the research group on "Noncrystalline solids and disordered systems" from I3N-Aveiro for all the help and assistance, especially to Nuno Ferreira, PhD.

\section{References}

1. World Health Organization, "World report on ageing and health," 2015, http://www.who.int/ageing/publications/world-report-2015/en/ (February 2017).

2. I. Korhonen, J. Parkka, and M. Van Gils, "Health monitoring in the home of the future," IEEE Eng. Med. Biol. Mag. 22(3), 66-73 (2003).

3. M. P. de Castro et al., "In-shoe plantar pressures and ground reaction forces during overweight adults' overground walking," Res. Q. Exercise Sport 85(2), 188-197 (2014).

4. T. Marasović, M. Cecic, and V. Zanchi, "Analysis and interpretation of ground reaction forces in normal gait," WSEAS Trans. Syst. 8(9), 1105-1114 (2009).

5. C. Kim and J. Eng, "Symmetry in vertical ground reaction force is accompanied by symmetry in temporal but not distance variables of gait in persons with stroke," Gait Posture 18(1), 23-28 (2003).

6. J. Perry, Gait Analysis: Normal and Pathological Function, p. 524, Slack, Inc., Thorofare, New Jersey (1992).

7. R. J. Abboud, “(i) Relevant foot biomechanics," Curr. Orthop. 16(3), 165-179 (2002).

8. E. Morag and P. R. Cavanagh, "Structural and functional predictors of regional peak pressures under the foot during walking," J. Biomech. 32(4), 359-370 (1999).

9. C. Bayón et al., "Locomotor training through a novel robotic platform for gait rehabilitation in pediatric population: short report," J. NeuroEng. Rehabil. 13(1), 98 (2016).

10. A. Razak et al., "Foot plantar pressure measurement system: a review," Sensors 12(7), 9884-9912 (2012).

11. T. Sim et al., "Predicting complete ground reaction forces and moments during gait with insole plantar pressure information using a wavelet neural network," J. Biomech. Eng. 137(9), 091001 (2015).

12. P. Roriz et al., "From conventional sensors to fibre optic sensors for strain and force measurements in biomechanics applications: a review," J. Biomech. 47(6), 1251-1261 (2014).

13. D. J. Webb et al., "First in-vivo trials of a fiber Bragg grating based temperature profiling system," J. Biomed. Opt. 5(1), 45-50 (2000).

14. P. Antunes et al., "Biaxial optical accelerometer and high-angle inclinometer with temperature and cross-axis insensitivity," IEEE Sens. J. 12(7), 2399-2406 (2012).

15. C. Leitão et al., "Feasibility studies of Bragg probe for noninvasive carotid pulse waveform assessment," J. Biomed. Opt. 18(1), 017006 (2013).

16. N. Alberto et al., "Optical fiber technology for eHealthcare," in Handbook of Research on ICTs and Management Systems for Improving Efficiency in Healthcare and Social Care, pp. 180-200, IGI Global, Hershey (2013).

17. N. Alberto et al., "Three-parameter optical fiber sensor based on a tilted fiber Bragg grating," Appl. Opt. 49(31), 6085-6091 (2010).

18. G. Wehrle et al., "A fibre optic Bragg grating strain sensor for monitoring ventilator movements," Meas. Sci. Technol. 12(7), 805-809 (2001).

19. H. J. Kalinowski et al., "Application of fibre Bragg grating sensors in biomechanics," in Chapter in Trends in Photonics 2010, J. Canning, Ed., pp. 315-343, Research Signpost, Kerala, India (2010).

20. J. Z. Hao et al., "Design of a foot-pressure monitoring transducer for diabetic patients based on FBG sensors," in 16th Annual Meeting of the IEEE Lasers and Electro-Optics Society (LEOS '03), Vol. 1 (2003).

21. T. C. Liang, J. J. Lin, and L. Y. Guo, "Plantar pressure detection with fiber Bragg gratings sensing system," Sensors 16(10), 1766 (2016).

22. R. Suresh et al., "Development of a high resolution plantar pressure monitoring pad based on fiber Bragg grating (FBG) sensors," Technol. Health Care 23(6), 785-794 (2015).

23. S. C. Wearing et al., "A comparison of gait initiation and termination methods for obtaining plantar foot pressures," Gait Posture 10(3), 255-263 (1999).

24. S. P. Silva et al., "Cork: properties, capabilities and applications," Int. Mater. Rev. 50(6), 345-365 (2005).

Maria Fátima Domingues received her $\mathrm{PhD}$ in physics engineering from the University of Aveiro, Portugal, in 2014 and she is currently 
a research fellow at the Instituto de Telecomunicações-Aveiro, I3NPhysiscs Department, University of Aveiro, Portugal and Consejo Superior de Investigaciones Científicas (CSIC)-Madrid, Spain holding postdoc grant from the Fundação para a Ciencia e a Tecnologia (FCT-Portugal). Her current research interests include new solutions of optical fiber sensors and its application in robotic exoskeletons and e-Health.

Cátia Tavares received her master degree in physics engineering from the University of Aveiro, Portugal in 2016. She is currently working as a researcher fellow at the Instituto de TelecomunicaçõesAveiro and I3N-Physiscs Department, University of Aveiro, Portugal. Her current research interests include de development of optical fiber sensors for eHealth solutions and its application in physical rehabilitation.

Cátia Leitão received her MS degree in biomedical engineering, specialization in biomedical instrumentation and biomaterials, from University of Coimbra in 2010. From 2010 to 2012 she was research fellow, and from 2013 to 2016, PhD student in physical engineering in University of Aveiro. Currently, she is a postdoctoral researcher at I3N \& Physics Department of University of Aveiro, Portugal. Her main research interests are optical fiber sensors applied to noninvasive cardiovascular monitoring, mainly in arterial pulse waveform acquisition and its implications on cardiovascular risk assessment.

Anselmo Frizera-Neto received his BS degree in electrical engineering from the Federal University of Espirito Santo UFES, Brazil, in 2006 and his $\mathrm{PhD}$ in electronics from the Universidad de Alcalá, Spain, in 2010. From 2006 to 2010, he was a researcher with the Bioengineering Group, Spanish National Research Council. He is currently a lecturer and researcher at the Electrical Engineering Department, UFES. His research interests include rehabilitation robotics, human-machine interaction, optical fiber sensors and movement analysis.

Nélia Alberto graduated with the degree in physics and chemistry from University of Aveiro, Portugal, in 2005. She received her PhD in physics from the same university in 2011. She is currently working as a postdoctoral researcher at the Instituto de Telecomunicações and Centre for Mechanical Technology and Automation in Aveiro. Her main research interests include the study, development and application of optical fiber based devices for sensing applications.

Carlos Marques received the $\mathrm{PhD}$ in physics engineering from the University of Aveiro, Portugal, in 2013. He was a Marie Curie fellow in the AIPT, Aston University, Birmingham, U.K from 2014 to 2016. Currently, he is a research fellow in the Instituto de Telecomunicações and Physics Department \& I3N, University of Aveiro, Portugal. He authored or coauthored more than 85 journal and conference technical papers with emphasis on optical communications and sensing using optical fiber technology.

Ayman Radwan received his PhD from Queen's University, Canada, in 2009 . He is a senior research engineer (Investigador Auxiliar) with the Instituto de Telecomunicações. He is mainly specialized in coordination and management of EU funded projects. He participated in the coordination of multiple EU projects. He is currently the project coordinator of CELTIC+ project "MUSCLES", as well as participating in the coordination of the ITN-SECRET. His research interests include IoT, 5G, and green communications.

Jonathan Rodriguez received his masters degree in electronic and electrical engineering and PhD from the University of Surrey UK, in 1998 and 2004 respectively. Since 2005, he is a researcher at the Instituto de Telecomunicacoes, Portugal. He is author of more than 300 scientific works, and his professional affiliations include: senior member of the IEEE and chartered engineer (CEng) since 2013, and fellow of the IET (2015).

Octavian Postolache is assistant professor at ISCTE-Lisbon University Institute and senior researcher at Instituto de Telecomunicacoes. He received his $\mathrm{PhD}$ in 1999 in electrical engineering from "Gh. Asachi" Technical University of lasi and the habilitation from Instituto Superior Tecnico, Lisbon, in 2016. He is an IEEE IMS distinguished lecturer, IEEE IMS Portugal chair and IMS TC-13 chair. His fields of interests are smart sensors for biomedical and environmental applications, pervasive computing for healthcare, WSN and IoT, and advanced signal processing for biomedical applications.

Eduardo Rocon received his PhD from the Universidad Politécnica de Madrid in 2006. His research activity was awarded with the Georges Giralt PhD award as the best PhD robotics thesis in Europe and the EMBEC scientific award. He is current a tenure researcher at the Bioengineering Group at the Consejo Superior de Investigaciones Científicas (CSIC). His research interests include rehabilitation, neurophysiology, biomechanics, adaptive signal processing, and human machine interaction.

Paulo André received his $\mathrm{PhD}$ in physics, and the Agregação title (habilitation) degree from the Universidade de Aveiro, Portugal, in 2002 and 2011, respectively. He joined the Instituto Superior Técnico, University of Lisbon, in 2013, as an associate professor, lecturing on telecommunications. He is an IEEE senior member and his research interests include the study /simulation of photonic and optoelectronic components, optical sensors, integrated optics, photonic graphene applications, multiwavelength optical communications systems, and passive optical networks.

Paulo Antunes received his $\mathrm{PhD}$ in physics engineering from the Aveiro University, Portugal, in 2011. He is currently an assistant researcher at the I3N-Aveiro (Institute of Nanostructures, Nanomodelling and Nanofabrication), he is also with the University of Aveiro Physics Department and the Instituto de Telecomunicações. His current research interests include the study and simulation of fiber Bragg gratings, data acquisition, optical sensing solutions for static and dynamic applications, including medical and structural monitoring. 